This item was submitted to Loughborough's Research Repository by the author.

Items in Figshare are protected by copyright, with all rights reserved, unless otherwise indicated.

\title{
Evaluating the impacts of working towards the International Safeguards for Children in Sport
}

PLEASE CITE THE PUBLISHED VERSION

https://doi.org/10.1016/j.smr.2019.05.009

\section{PUBLISHER}

Elsevier (C Sport Management Association of Australia and New Zealand

VERSION

AM (Accepted Manuscript)

\section{PUBLISHER STATEMENT}

This paper was accepted for publication in the journal Sport Management Review and the definitive published version is available at https://doi.org/10.1016/j.smr.2019.05.009

LICENCE

CC BY-NC-ND 4.0

\section{REPOSITORY RECORD}

Rhind, Daniel, and Frank Owusu-Sekyere. 2019. "Evaluating the Impacts of Working Towards the International Safeguards for Children in Sport”. Loughborough University. https://hdl.handle.net/2134/38051. 
Evaluating the impacts of working towards the International Safeguards for Children in Sport

\begin{abstract}
In this paper, the authors evaluate a global strategy to safeguard children against abuse in sport. The experiences of people within 32 of the organisations who were working towards the International Safeguards for Children in Sport were captured over a two-year study. At the organisational level, self-audits demonstrated that progress was made during the project by deliverers (who worked directly with children) from having $45 \%$ to $64 \%$ of the Safeguards fully in place. Governors (who governed other organisations which worked directly with children) improved on the same figure from $25 \%$ to $53 \%$. Progress was also identified using the concept of Activation States through in-depth interviews at the start and end of the project. Positive changes were found at the personal level with respect to people's feelings, knowledge and behaviours related to safeguarding as well as how safeguarding is discussed in the organisation. Group discussions also revealed changes with respect to how children, coaches, parents and the broader community were behaving with respect to safeguarding. An increase in the number of disclosures was also identified as an important impact of the project. The International Safeguards for Children in Sport are now endorsed by 125 organisations who work with a total of over 35 million children. The implications of these findings are discussed along with the future directions of work in this area.
\end{abstract}

Keywords: abuse; violence; welfare; well-being; children's rights 


\section{Introduction}

Based on a meta-analysis, Stoltenborgh, Bakermans-Kranenberg, Link and van Ijzenboorn (2015) concluded that "Child maltreatment is a widespread, global phenomenon affecting the lives of millions of children all over the world, which is in sharp contrast with the United Nation's Convention on the Rights of the Child." (p.37). The context of sport is not immune to this broader social problem. High profile cases in the media along with research over the past 25 years has highlighted a wide range of welfare concerns at the individual, relational and organisational levels (Brackenridge, 2001; Brackenridge, \& Rhind, 2014; Mountjoy, Rhind, Tiivas, \& Leglise, 2016; Rhind, McDermott, Lambert, \& Koleva, 2014). Research has also highlighted that unhealthy practices can be normalized and tolerated within sport (Alexander, Stafford, \& Lewis, 2011; Papaefstathiou, Rhind, \& Brackenridge, 2012). In response, strategies have been developed and implemented to prevent and manage abuse in sport. We report an analysis of the impacts of the International Safeguards for Children in Sport (referred to as the Safeguards from now on) based on the experiences of people across 32 organisations.

\subsection{The International Safeguards for Children in Sport}

The state of the art review of violence prevention programmes commissioned by UNICEF focused on industrialized countries (Brackenridge, Kay, \& Rhind, 2012) identified that the existing body of work at that time had primarily been conducted in single countries typically based in the global north. The review thus called for a global approach and highlighted the need for a set of international standards for safeguarding in sport. To address this identified need, a working group with representatives from a range of influential organisations was established in 2012 to consider how sports provision could be kept safe. This working group developed a draft set of child safeguarding Standards (please note that, based on feedback 
during the research process, the final name was changed from Standards to Safeguards). The safeguards set out the actions that all organisations working in sport should have in place to ensure children are safe from harm (Mountjoy et al., 2016). They reflect international declarations, the United Nations Convention on the Rights of the Child, relevant legislation, government guidance, existing child protection/safeguarding standards and good practice.

The development of these International Safeguards was informed by a comprehensive review of the extant literature (Brackenridge et al., 2012). This included empirical evidence, relevant policies and existing local or national programmes (Brackenridge et al., 2012). These International Safeguards help to overcome the range of challenges that have been highlighted in the literature related to safeguarding in sport. Firstly, there is a need for a global framework which can guide and facilitate the development of policies, procedures and practices at the national and local level (Chroni et al., 2012). Secondly, they also encourage organisations in sport to adopt proactive measures to prevent abuse, such as background checks and education. Thirdly, they help organisations to implement responsive measures when a safeguarding concern is disclosed. Finally, they encourage organisations to embed evidence-based practice through effectively collecting and learning from data (Raakman, Dorsch, \& Rhind, 2010).

The International Safeguards for Children in Sport are:

1. Developing Your Policy

2. Procedures for Responding to Safeguarding Concerns

3. Advice and Support

4. Minimizing Risks to Children

5. Guidelines for Behaviour

6. Recruiting, Training and Communicating 


\section{Working with Partners \\ 8. Monitoring and Evaluating}

In a recent audit, 125 organisations have now endorsed the Safeguards, and these organisations as a whole work with a total of over 35 million children.

\subsection{Theoretical Foundations}

This project was grounded in the theoretical foundations of Bandura's (2001) work on the social cognitive approach and the Reciprocal Determinism Theory. This has been developed and applied to the promotion of safer behaviour within organisations by Cooper (2000) through the Reciprocal Safety Culture model. It suggests that safety culture is developed and maintained through interactions between organisational, personal and behavioural factors.

Organisational factors concern the environment in which the safety behaviours are enacted. This is shaped, for example, by relevant policy, procedures and resources. Personal factors are related to individuals' values and beliefs which influence safeguarding behaviours. This relates to how people talk about safeguarding, their knowledge of the topic and their feelings towards safeguarding. Behavioural factors concern the observable safeguarding-related actions enacted by people within the organisation.

Researchers have found that a positive and strong safety culture can enhance safety performance (Edwards, Davey, \& Armstrong, 2013). This has been illustrated in diverse industries such as construction, aviation and healthcare (Edwards et al., 2013). However, whilst the concept of safety culture has received significant attention in a range of high-risk industries, it has not yet been systematically applied to the safeguarding of children. This is an innovative contribution of this present study with Cooper's 2000) Reciprocal Safety Culture model under-pinning the development, implementation and the evaluation of the Safeguards. Cooper (2000) argued that the dynamic nature of this approach allows for the 
consideration of human and organisational contexts, while also allowing the triangulation of methodologies and multi-level analyses of safety culture. The ways in which this theoretical approach informed the methods used in the evaluation is explained in the next section.

\subsection{The need for evaluation}

Without data, it is not possible to have an accurate impression as to whether any progress is actually being made. The continued development of the Safeguards should thus be based on robust evidence which enhances understanding of how their effective and efficacious implementation can be maximised. Speaking more generally about safeguarding systems, Wessells (2009, p. 12) explained:

A stronger evidence base is needed for the purposes of building inter-agency standards of practice and harmonising supports at national and international levels...the sector needs a stronger evidence base that enables inter-agency consensus on effective practice, and that serves as a foundation for good practice guidelines that harmonise diverse child protection efforts.

There is a sizeable and rapidly growing literature about programme evaluation. Current thinking encourages researchers to address both the process and outcomes of a change programme and not to focus solely on outcomes (Coalter, 2007). As a result, the broader evaluation was conducted longitudinally, and ensured that data were collected at the start and end of the project as well as periodically throughout the process of implementing the International Safeguards. This is in line with the recommendations of researchers who have called for such an approach in evaluative social research to overcome the potential limitations of collecting data at one moment in time (Brackenridge et al., 2005). People's attitudes towards safeguarding are likely to fluctuate over time and hence a longitudinal approach can help to capture the direction and intensity of any such changes. It also assumes that any change will not necessarily be linear or causal in nature. Conducting research over a longer 
period of time also allowed the researchers to build rapport with the participants through ensuring that it was genuinely a collaborative project at each stage of the process. The process evaluation has previously been published (Rhind, Kay, Hills, \& Owusu-Sekyere, 2017). We report the outcome elements of the evaluation.

\subsection{Aims}

The aim of the broader research project was to answer the overarching research question: are the Safeguards appropriate for global adoption to keep children safe while participating in sport?" We focus on the perceived impacts of working towards the Safeguards and therefore address a significant gap in the literature in terms of evaluating the first project to develop and implement a global approach to safeguarding children in sport. Such work is important due to the millions of children who participate in sport around the world. The specific research questions were:

Research Question 1: What was the perceived progress towards the Safeguards? (organisational factors)

Research Question 2: What was the perceived impact of the Safeguards on people's voices, knowledge and feelings? (personal factors)

Research Question 3: What was the perceived impact of the Safeguards on observable actions? (behavioural factors)

Research Question 4: Was there evidence of reciprocity between the organisational, personal and behavioural factors?

\section{Method}

\subsection{Participants}

Ethics approval was granted by the University's ethics Committee prior to participant recruitment. Each of the pioneer organisations $(n=49)$ who had volunteered to pilot the 
Safeguards was contacted to provide an overview of the aims and nature of the research. An informed consent form was then sent to any organisations $(n=32)$ which showed an interest in taking part in the research. This represented a response rate of 64\%. All interested organisations $(n=32)$ signed and return this form. All participants were informed of their right to withdraw at any time and that their data would remain anonymous and confidential.

Initial discussions with the participants at the first group meeting within a conference highlighted that the different types of organisation may have different experiences due to the nature and focus of their work. Two broad categories were identified in consultation with the participants. The first of these were categorized as Deliverers $(n=16)$ and this included organisations which work directly with children (e.g., sport clubs, sport for development programmes and academies. These organisations were based in Africa $(n=7)$, Asia $(n=4)$, Europe $(n=3)$, North and South America $(n=2)$. The second group were categorised as Governors. These organisations work with partner or member organisations who in turn work directly with children (e.g., Governing bodies, Government agencies or funding organisations). Governors worked internationally $(n=11)$, Europe $(n=3)$ or in North and South America $(n=2)$. In order to maintain anonymity, the details given regarding the participants and their organisations are limited.

An important indicator of change is the experience of key change agents. The research team therefore identified the key change agent within each organisation who had overall responsibility for safeguarding children and the implementation of the Safeguards. These participants represented a range of different roles including Chief Executives, Directors, Managers, Safeguarding Officers and Coaches. It was important to understand their experiences as these participants were in a position to facilitate and symbolise the implementation of the International Safeguards. It was also important to build rapport between the researchers and the participants and to maintain an effective working partnership 
throughout the project. This was initiated through a face-to-face workshop with the participants at the start of the project and at the ends of years 1,2 and 3. These face-to-face interactions were supported by regular communications via e-mails, video calls and online discussions.

\subsection{Data collection}

Three methods were used to collect data related to the dimensions of Cooper's (2000) underlying Safety Culture model (i.e., organisational, personal and behavioural factors). First, we employed the Self Audit Tool, which facilitates self-reflection through respondents considering the extent to which their organisation has made progress towards each aspect of the Safeguards. Within each Safeguard, the relevant criteria are displayed. Respondents are asked to decide if their organisation has each criterion in place (A), partially in place (B), or not in place (C). Each participant completed the Self Audit Tool for their organisation at the start of the project and again at the end. This provided basic descriptive data which indicated the extent to which progress had been made towards each Safeguard.

In addition, we formally interviewed each participant on two occasions. The first interview took place between 6-12 months of the project with the second interview being conducted between 30-36 months. An interview schedule was developed based on the concept of Activation States proposed by Brackenridge et al. (2005). This approach is explained in more detail within section 2.3.2 below. The interview explored how the participant was experiencing the culture of their organisation with reference to the different dimensions of the Activation States framework (i.e., voices, knowledge and feelings). For example, participants were asked: "how do people talk about safeguarding within your organisation (voices)," "what do people know about safeguarding in your organisation (knowledge)," and "how do people feel about safeguarding in your organisation (feelings)?" The interviews were flexible 
"as appropriate to the emergent relationship that is formed between the interviewer and the interviewee" (Patton, 2015, p.433). For this reason, we used a semi-structured approach. As a result, the interviews had the orderliness of the structured interview type, in the form of a prescribed set of questions. However, we were not limited to asking those questions in a strict order and modified the content and order of questions based on perceptions of what seemed most appropriate during the dialogue. Furthermore, we asked follow-up questions and deviated from the interview guide, making it a truer social meaning making act (Sparkes \& Smith, 2014). Interviews were conducted via video call or telephone due to the global geographic spread of the participants. They ranged in length from 45-105 minutes.

Finally, we had group discussions. These discussions took three different forms. Firstly, organisations were allocated into one of nine Virtual Learning Sets (VLS). Organisations were grouped based on their mission (e.g., competitive sport, participation), size (e.g., international governors, local deliverers) and geographical location to ensure that Skype calls could take place at convenient times for all participants. Each VLS was co-ordinated by a member of the International Safeguards Working Group. The VLS co-ordinator had experience relevant to safeguarding children in the context in which their group's organisations were operating (e.g., elite sport, sport for development etc.). These learning Sets were conducted approximately every three months via video call to discuss experiences and share good practice. The coordinators of these sets completed a template form after each discussion to share the key messages. Secondly, a password-protected on-line forum was also created and maintained throughout the data collection period. This provided all participants an opportunity to share their thoughts or pose questions to the other pilot organisations at any time. Finally, workshops were arranged at the start of the project and at the end of years 1,2 and 3. Each meeting began with a presentation from the research team. This was followed by small group discussions with the key points then being fed back to the group as a whole. The 
research team made notes on these discussions which then fed into the on-going analysis of the project.

Group discussions in these different formats were utilised to explore any behavioural changes observed by the participants with respect to the key stakeholders in their organisations (e.g., staff, parents, coaches and children). This group approach was selected to enable more naturalistic discussions (Marshall \& Rossman, 1999). They were also designed such that the participants could learn from each other and hence experience the research project as an enriching experience as opposed to the typical approach which extracts data from individuals with the process having limited benefits for them.

Group discussions also have the benefit of promoting synergies between the participants which can stimulate experiences which may not be identified through other methods (Lindlof $\&$ Taylor, 2002). Finally, group discussions are appropriate when discussing sensitive topics as participants can feel supported by the presence of others sharing similar experiences (Tracy, Lutgen-Sandvik, \& Alberts, 2006).

\subsection{Data Analysis}

We analysed the data with respect to the three dimensions of Cooper's (2000) Safety Culture model and potential interrelationships among the dimensions. First, we assessed organizational changes using the Self-Audit Tool data. The overall percentage of the criteria for a given Safeguard which were viewed as being 'in place' were calculated for the

Deliverers and Governors. For example, there are eight criteria for Safeguard 1 (Developing Your Policy). There were 16 Deliverer organisations which created a total of 128 ( $8 \times 16)$ criteria. If 32 of the criteria were in place across the 16 organisations then this was calculated as an overall percentage being in place of $25 \%$ (i.e., $32 / 128$ ). These percentages were then compared to identify the extent to which any progress had been reported. These data provided 
an indication of the organisational factors in terms of whether any changes had been observed in the broader context of safeguarding in the organisation (Cooper, 2000).

We also assessed personal changes. In order to assess personal changes, a deductive approach to data analysis was adopted through using the concept of Activation States as a guiding framework (Brackenridge et al., 2005). This approach provides an indication as to how 'active' people are with respect to safeguarding. Each transcript was reviewed with respect to three of the dimensions in Brackenridge et al.'s (2005) framework: Voices (i.e., what people say about the Safeguards), Knowledge (i.e., what people know about the Safeguards) and Feelings (i.e., how people feel about the Safeguards).

For each of these dimensions, each organisation was categorised into one of the following five Activation States: Opposed (e.g., critical of the need for the Safeguards); Inactive (e.g., demonstrating no awareness related to the Safeguards); Reactive (e.g., demonstrating reluctant compliance with the Safeguards); Active (e.g., demonstrating appropriate awareness related to the Safeguards) or Proactive (e.g., demonstrating full commitment and advocating for the Safeguards). As an example, a participant could report that the general culture within their organisation is characterised by people talking negatively about safeguarding (voicesopposed), show a lack of awareness (knowledge-inactive) and have negative emotions towards safeguarding (feelings-opposed).

During a team meeting, we developed and revised the procedures to be used to analyse the data. Following the approach outlined by Brackenridge et al., (2005), we read a sample of transcripts and then independently identified the salient Activation States through completing a grid showing opposed-proactive on the horizontal axis and the dimensions on the vertical axis. We assigned the same categorisations on $94 \%$ of occasions, which represents an acceptable indication of inter-rater reliability (Brackenridge et al., 2005). 
The transcripts from the in-depth interviews at the start and end of the research project with the 32 key change agents were analysed with respect to perceptions of the predominant organisational culture. The identification of Activation States affords a clear visual representation of the state ('profile') of each organisational culture. The modal Activation State for the group of Deliverers and Governors was identified at the start and end of the project. This helped to give an indication as to where any changes had been experienced. An organisation whose profile matched the broader profile of the deliverers and governors was then identified. This ensured that participant quotes from these organisations could be highlighted to illustrate the changes which have been observed. This qualitative data thus helped to exemplify the broader changes in the Activation States profiles of the deliverers and governors. The concept of Activation States was used because it has been found to be an effective way of subtly exploring the psychosocial dimensions of an organisation's culture of safeguarding (Hartill, Lang, \& Ashley, 2014).

Third, we assessed the behavioural changes using the interview data. A deductive approach was employed through applying the concept of Activation States, using similar procedures to those explained in the previous section (Brackenridge et al., 2005). However, this analysis focused on the Activation State with respect to the observable actions that people were taking in relation to safeguarding. As previously noted, the five categories were used: Opposed (e.g., actions which hinder the implementation of the Safeguards), Inactive (e.g., taking no actions related to the Safeguards), Reactive (e.g., taking actions but only in response to persuasion), Active (e.g., taking appropriate actions in line with the Safeguards), or Proactive (e.g., taking actions related to the Safeguards which go above and beyond those required by the given role). The profile of Activation States was determined at the start and end of the project for each individual organisation. The modal profile was also identified for the deliverers and governors along with illustrative quotes. 
We also examined reciprocity. In relation to the various group discussions, all of the qualitative data was transcribed verbatim and analysed using the stages of thematic analysis outlined by Braun and Clarke (2006). This analysis explored how the different dimensions in Cooper's (2000) Safety Culture model interacted to create cultural change. An inductive approach was adopted for this aspect of the data analysis. Although Cooper's (2000) model underpinned the focus on organisational, personal and behavioural factors, this specific analysis explored the ways in which these factors interacted to facilitate changes in safety culture. As there was a lack of existing literature on this topic to enable a guiding framework to be developed with confidence, an inductive approach was employed. This enabled themes to emerge from the data without any constraints and this was viewed as the most appropriate approach due to the exploratory nature of this aspect of the research.

The first stage of this analytical process was familiarisation. In order to do this, we read through the transcriptions several times. At this stage, notes were taken, to be considered in more detail at a later stage. The second stage involved generating initial codes. We manually worked through printouts of each interview to determine both semantic and latent features of the data which potentially evidenced a reciprocal relationship between the organisational, personal and behavioural factors. The third stage began once all data had been coded and codes had been collated. We sorted through each of the codes attempting to place them into groups with codes which represented a particular aspect of reciprocity. This involved the researchers going to and from unsorted groups of codes, attempting to find the best groupings. We followed Braun and Clarke's suggestion that themes that do not fit anywhere may be temporarily categorised as miscellaneous (2006). Stage four involved the review and refinement of the identified themes. In stage 5, the researchers defined each theme. Braun and Clarke describe this as "identifying the essence of what each theme is about" (2006, p. 92). 
This required the researchers to interpret the codes, rather than merely paraphrase the data, and they relied upon the notes developed in stage two as codes were initially developed.

\subsection{Trustworthiness}

We were part of the International Safeguards Working Group and hence were embedded in the project from the start and throughout. However, clear memorandums of understanding were signed by the researchers and all participants to clarify that we were independent of the broader Safeguards project. We had bracketing interviews at the start and periodically through the project to share expectations and assumptions such that these could be challenged and appropriate mitigation strategies could be implemented. A range of strategies was also employed to enhance the trustworthiness of the data analysis process. Participants were given opportunities to comment on the accuracy of the transcription as well as the interpretation of the data with regards to the emergent themes. Regular meetings were also held with the people leading each of the learning sets to ensure that their input was encouraged throughout the process of analysis.

\subsection{Findings and discussion}

The findings are presented with respect to each of the research questions.

3.1 What was the perceived progress towards the Safeguards (organisational factors)

Table 1 presents the percentages of the criteria in place for each of the Safeguards for the deliverer organisations. The data displays the status at the start and end of the project. The percentages reflect how many of the criteria were not in place, partially in place or fully in place. Safeguards 1 (Policy) and 5 (Guidelines) had the most criteria in place by the end of the project. It may be that developing a policy and guidelines are logical first steps and could be areas in which progress is more readily made relative to the other Safeguards. The rest of the Safeguards can then be grounded in this policy, which outlines the organisation's values 
and commitment towards safeguarding children. Links between the policy and a set of guidelines that clearly articulate the expected behaviours can also be emphasised.

Meaningful progress was also made by the deliverers with respect to monitoring and evaluation. There was a growing recognition of the value of having evidence to both inform and evaluate practice. Evidenced based communications were important when discussing the need for safeguarding with key stakeholders (e.g., parents, coaches, funders etc.). Monitoring and evaluation have been found to be important factors in developing a safety culture and facilitating behaviour change in other industries (Frazier, Ludwig, Whitaker, \& Roberts, 2013).

The areas with the fewest criteria in place for the deliverers were Safeguards 2 (Procedures) and 3 (Support). Fully implementing effective procedures and support is likely to take time and hence this may explain why further progress had not been made. It is also important to highlight that by the end of the project only $10 \%$ of the criteria for Safeguards 2 and 3 were viewed as having nothing in place. This suggests that some progress had been made, although there remained scope for these Safeguards to be further developed and embedded. It is also noteworthy that all of the deliverers had at least partially met the criteria in relation to working with partners. The collaborative nature of this project encouraged organisations to work more closely with relevant partners and these partnerships proved to be useful resources in facilitating progress towards other Safeguards.

*** Insert Table 1 near here***

Table 2 presents the percentages of the criteria in place for each of the Safeguards for the governor organisations. Safeguards 1 (Policy) 6 (Communicating) and 7 (Partnerships) had the most criteria in place by the end of the project. The progress made by the Governors with respect to having a policy is particularly notable. As these organisations did not directly work 
with children, the relevance of having their own safeguarding policy was not always acknowledged. However, the fact that all of these organisations had a policy in place by the end demonstrates that this was seen as an important step forward. A safeguarding policy was a mechanism through which the governors could demonstrate a commitment to safeguard children. Embedding effective mechanisms to communicate with stakeholders about safeguarding was also a priority. The progress towards Safeguard 7 was also indicative of an important change in approach amongst these organisations from one of working in isolation to one of working in partnership. It was often through considering the items in Safeguard 7 that participants were able to make progress with respect to criteria in other Safeguards.

The areas with the fewest criteria in place for the governors were Safeguards 2 (Procedures), 3 (Support) and 4 (Risk). As explained for the deliverers, fully implementing procedures and support services to safeguard children is likely to take time. Furthermore, the concept of identifying and mitigating risk through a proactive approach is significantly different from the typical approach that focuses on responding to concerns. Such a change may take time to become fully embedded. Moving towards a proactive approach through the use of risk assessments has been found to be an effective strategy for developing a safety culture in other industries (Frazier et al., 2013). It is noteworthy that there were significant reductions in the criteria across these three Safeguards with nothing in place and hence some progress was reported.

***Insert Table 2 near here***

In terms of the first research question, overall the deliverers improved from 45 to $64 \%$ and the governors improved from 25 to $53 \%$. This demonstrates that changes were reported with respect to the broader environment created by the organisation. 
These organisational factors represent one of the 3 key dimensions in Cooper's (2000) model of safety culture. This model would therefore suggest that these environmental changes should help to develop a safeguarding culture (Fernández-Muñiz, Montes-Peón, \& VázquezOrdás, 2007). However, it is important to emphasise that organisational factors in isolation, such as having a policy, are unlikely to make a significant cultural change. As highlighted by a Lead Welfare Officer in a study conducted by Hartill and Lang (2014, p. 621): “there's more to it than having a policy." Jacobs, Smits and Knoppers (2016) also argued that sporting organisations tend to make the false assumption that the existence of a policy alone will keep children safe. This is despite the lack of evidence to substantiate this claim (Vertommen et al., 2016).

There is a tendency for safeguarding crises to be linked to organisational factors, such as the absence of policy, and the development of codes of conduct and policies is a typical response when such crises occur in sport (Brackenridge \& Rhind, 2014). The key point is that through effective implementation, these broader environmental changes can influence the personal and behavioural factors and hence contribute to a genuine and sustained change in safety culture.

3.2 What was the perceived impact of the Safeguards on people's voices, knowledge and feelings? (personal factors)

For the deliverers, the ways in which people talked about safeguarding (voices) were found to change from opposed to active throughout the project. Some of the participants experienced resistance when safeguarding was viewed as being forced upon them by an external pressure. For example, one participant explained: 
People are against this kind of work when it is talked about as coming from the attitude of 'we come from the empire and we know what is best for you. This leads to people talking negatively about the need for safeguarding.

By the end of the project, this same participant reported a significant change:

Since starting the Safeguards project, we have gone from a position of people saying that they cannot believe you are making me do this to one where they say I would not put my child in a club that did not do this.

The profile for people's feelings also changed from opposed to active. There was some initial opposition based on a perception that safeguarding was not required in the given context. In one organisation, this was related to social norms, for example:

Scolding children is not seen as abuse. Having a child run around a field 5 times is not a problem. We are coming from a culture in which these are seen as ok. These are the feelings which we have to engage with.

The same participant observed a significant change:

I can now say that we get much less resistance. When we get new people there may be some questions as they have just not come across safeguarding before. Everyone else is much more invested and they see how important it is.

With respect to knowledge, the profile changed from inactive to active. This is illustrated by one participant, who identified a lack of knowledge in the organisation at the start of the project, "at the moment, we have a policy and procedures on paper but there is a lack of awareness of these amongst our staff and the children themselves so we will focus on improving this through the project." By the end of the project, a change in relation to people's knowledge related to safeguarding was observed and this was associated with changes in 
behaviour: "...we would finish meetings and girls would be going home, some of them late in the dark. Now we know that at $3 \mathrm{pm}$ we have to finish so everyone has enough time to get home before its dark."

These pairs of quotations illustrate a broad change in terms of the personal factors for the Deliverer organisations. Overall, participants perceived that people now spoke and felt more positively about safeguarding. People were now viewed as having the knowledge to fulfil the safeguarding elements of their role. The changes identified in the activation states therefore suggest that changes had been observed in relation to the voices, feelings and knowledge of people within the deliverer organisations.

Significant changes were also identified across all areas of the activation states for the Governor's. The modal response for the voices dimension changed from opposed to reactive. At the start of the project, one participant explained that the opposition was linked to workload: "Even though we try to integrate it, people still see it as extra and as an additional segment. They say here we go again with safeguarding. I have 10,000 other things to do.” The same participant illustrated the broader change to a reactive profile for the Governor organisations. It remained reactive because people had started to engage in discussions about safeguarding but only in response to potential risks being highlighted:

The argument that we should do it because it is the right thing to do for children does not work. What does work is when we point out that you are growing your game and that there is a potential reputational risk. This gets people to listen.

The Governors profiles with respect to feelings also changed from opposed to active. This is illustrated by one participant as follows: 
In the 3 years of the Safeguards project we have gone from people at the governing body level thinking that this is not our business to now where it is mandated as part of our strategic plans moving forward, this has been a great change. We have also been given time to work through the self-audit with our member organisations in $25-30$ countries. In many cases there is nothing yet in place but at least this is a start to the journey. And people agree it is important.

A similar journey was experienced by a participant working with coaches:

At the beginning people just saw it as hoops that they had to jump through. This was particularly from our coaches, who are mostly volunteers, and they saw it as them having to do this and do that to tick boxes. People have really started to embrace safeguarding. This has come about through us building trust with our coaches and members.

The knowledge profile was perceived to change from inactive to active for the Governor organisations. For example, at the start of the project one participant explained: "Our people are experts on the topics and on sport for development, but they will not necessarily have the expertise to manage disclosures and maybe they would feel inadequate to help out with those kinds of things." At the end of the project, the same participant reported: "Our people are now aware of our policies and procedures. They know that everyone has a role with respect to safeguarding and they would know what to do if any concerns were disclosed."

These quotations illustrate a broad change in terms of the personal factors for the Governor organisations. Participants perceived that people across these organisations spoke and felt more positively towards safeguarding and that colleagues now had the knowledge to fulfil the safeguarding elements of their role. Some aspects of the profile were reactive in that people would engage with safeguarding when it was associated with a key motivating factor (e.g., 
reputation, linked to funding). It can thus, be concluded that the data suggest that a change in the Activation States within the Governor organisations had also been observed.

Cooper (2000) highlights personal factors as key dimensions of the model of safety culture and these have been shown to play a key role in influencing safety performance (Christian, Bradley, Wallace, \& Burke, 2009). The current research supports existing literature suggesting that the ways in which an organisation engages with people are important in terms of shaping how those people feel, think and talk about safety (Cox \& Cheyne, 2000). As a result, it is important to engage with people's voices through using discourses that are meaningful to them, engage with feelings through highlighting relevant motivating factors and to engage with people's knowledge through being clear about exactly what they need to know to fulfil their role successfully.

3.3 What was the perceived impact of the Safeguards on observable actions? (behavioural factors)

The Activation States for the Deliverers with respect to the behavioural dimension changed from inactive to active during the project. This change is exemplified by the experience of one participant within a sport for development organisation for girls. At the start of the project this participant explained that:

Children come from families where they don't always have a voice. So to be able to voice your opinion at the age of 13 against someone who is older than you, that is not traditionally or culturally, that is not done. So encouraging those kids at 13, 14, 15 to go and speak to the social worker to report inappropriate behaviour by an adult, that has been quite tricky because the cultural practice is that this person is older than me, I have to respect them, I cannot disrespect them. To report against them would be seen as disrespecting an elder. 
By the end of the project, the same participant reported that observable change had occurred. This was particularly in relation to the empowerment of the girls through the introduction of peer leaders. During training camps for peer educators, each class had an assigned Peer Welfare Lead. This person was responsible for helping to ensure that the girls' rights were not violated during the camp. The Peer Welfare Lead was also a designated person to whom concerns could be reported should the girl not want to discuss the matter with a member of staff. This approach helped to safeguard the girls through engaging other girls within the safeguarding system. This Participant also highlighted the importance of having alternative avenues through which the girls can disclose concerns:

You know, once the girl is not ready to share with the field leader she can share with the team captain who can sit with the committee and share the information....and this has led to a genuine change in behaviour.

The Activation States for the Governors with respect to the behavioural dimension changed from inactive to reactive during the project. At the start of the project, one participant explained how a lack of incentives can contribute to inactivity: "If I am a national governing body, why would I want to operate with such standards? Why would I want to have a policy in place, especially if I am still getting funding without it?"

By the end of the project, observable change was reported but this was often in response to another pressure and hence it was illustrative of a reactive approach. A participant said:

In terms of child protection, I think that it is always a bit imposed from the top. Of course, we discussed our policy with the staff and the girls but if it had not been for our donors pushing for it then it probably would not have happened. 
The Deliverers and Governors were therefore found to be inactive in relation to safeguarding at the start of the project. By the end of the project, changes had been observed suggesting that deliverers were now adopting an active approach whilst governors were found to be more reactive. Overall, working towards the International Safeguards was perceived to have impacted people's behaviours such that they were more active with respect to safeguarding children.

Behavioural factors are the third dimension in Cooper's (2000) model of safety culture. This research highlights the importance of considering how people are encouraged and supported to enact safety-related behaviours. In line with Cooper's (2000) model, people need to be incentivised to safeguard children in sport and this reflects the research findings from other domains like oil manufacturing (Filho, Andrede, \& Marinho, 2010) and construction (Choudhury, Fang, \& Mohamed, 2007a). Clearly, the safeguarding of children is fundamentally different from the safeguarding of property, products or employees. Sport often involves working with volunteers rather than paid employees. This ensures that the kinds of targets and financial incentives used in other industries are not appropriate when considering children in sport. There is thus a need to find alternative strategies to incentivise people to safeguard children in sport as well as to remove any barriers that may hinder the enactment of safety-related behaviours.

3.4 Was there evidence of reciprocity between the organisational, personal and behavioural factors?

The data regarding the organisational, personal and behavioural factors were presented separately in the above sections. However, in line with Cooper's (2000) Safety Culture model, it is important to emphasise that these three dimensions interact to develop and maintain a culture of safeguarding within sport. Four examples are now outlined with respect 
to the ways in which the organisations engaged with children, coaches and the broader community. An increase in the level of disclosures was also highlighted as an important outcome. Each of these themes are now discussed and illustrated with participant quotes to demonstrate how these were facilitated by a reciprocity between the organisational, personal and behavioural factors.

\subsubsection{Engaging with children}

A Head of Safeguarding outlined an example of how this project had impacted practice in terms of how the organisation interacted with children. This impacted at the organisational level because there is a key criterion across all Safeguards which relates to listening to children. This organisation embedded this requirement throughout their decision-making procedures. The Head of Safeguarding highlighted that this needed to be a genuine engagement:

You see in a lot of organisations it's a tokenistic declaration where children are engaged but they are not active in the decision-making process and they are not actually contributing to the change because they are not given that opportunity. So, for us, in any work we do, we want to ensure that if we are asking children something, then they know where the information is going and what we are doing with it and how they themselves are actively contributing to positive change and decision making.

This participant went on to give the following example:

...when we were writing our disciplinary framework, they (the children) were involved, and they now own it. They understand it and so when it comes to a time when they are involved in that process, if they have breached our code of conduct, then they understand what will happen to them. They actually have more ownership 
of that because it is something that they have been involved in. So instead of the organisation forcing rules and activities on them, they are part of the process of development. It is really the Safeguards that have driven this because without this I would not have been able to demonstrate how we involve young people in decision making.

This suggests that the procedural change had impacted the children's knowledge regarding safeguarding (personal factor). This in turn was associated with a change in the children's behaviour. For example, for one event, children were observed making several points about the safety implications of particular decisions. This included points on the potential physicality of some games, the potential time spent standing in the sun and the likelihood of disputes arising from competitiveness. These concerns led to changes in the plans and procedures associated with the event. This example illustrates how organisational, personal and behavioural factors can interact to influence the culture around how people are engaging with children.

\subsubsection{Engaging coaches}

One organisation made significant progress in terms of how they communicated with their coaches regarding safeguarding (Safeguard 6). This organisation engaged with voices, knowledge and feelings (personal factors) through participatory learning.

Our learning model is not one of pouring information into people's heads. We consciously seek to encourage participatory learning. That leads to value judgements about a safe learning environment...it's not about coming in and saying well you must do this, instead we are working in partnership so we view and question their cultures and traditions. They have to buy in and say, yes this is important and is a good way to go forward, not just...well my dad hit me and I turned out fine. 
Through the project, the participant from this organisation discussed how they had observed changes in the ways in which coaches interacted with children. A significant reduction in incidents of corporal punishment was observed. This was perceived to be associated with an increase in the use of questioning techniques during interactions with children: “...they have changed the way they respond to children. So for example if there's a naughty child they are not so quick to punish, they try to understand their behaviour and cover all angles."

The training of coaches also impacted safeguarding behaviour outside of the organisation:

One way in which our involvement with the Safeguards has impacted children is through our provision of first aid. As part of the self-audit, we reviewed our injury log. We identified that the kinds of injuries being suffered were not really being addressed by our training for coaches. We adapted the curriculum to include foot and spinal injuries, which are both prevalent in our society. The coaches really liked this as they could transfer these skills back into the community, such as dealing with road traffic accident injuries. This may appear tangential to the Safeguards but it came about as a result of our involvement in this project and has helped to better safeguard the children in our organisation.

This example illustrates how organisational, personal and behavioural factors can shape the interactions between coaches and children. The key point was not merely the provision of training but rather the way in which the training engaged with the coaches and included discussion of topics which were meaningful to them. This combination of factors appeared to contribute to the observed changes in behaviour.

\subsubsection{Engaging the community}


Another organisation made progress with respect to how they engaged with the broader community (Safeguard 7). Working towards the Safeguards helped to safeguard children through raising awareness amongst this community. This was particularly important in terms of engaging with parents:

Since starting the project, we are now trying to create a more friendly atmosphere for the children. We are communicating with parents much better on what the children are going to be doing. Parents did not really have any concept of child protection before this project...parents now understand that participants engaged in our programs need consents signed for them to take part in our activities.

This was then perceived to have a broader impact: "The more they get into the culture that, you know, my child is receiving this level of protection, the more it begins to leak into their personal lives". This in turn was perceived to impact parental behaviour: "It has actually proved a good marketing tool. Parents now look to see if the safeguards are in place before letting their children join".

This illustrates the argument that effective safeguarding is not just about preventing negative outcomes, but it also has potentially significant positive outcomes for an organisation, such as in relation to increased participation rates. The impact also extended to key figures within the community. For instance, one participant described the following case:

We had an issue in one community where a young girl had been sexually abused by her brother. Usually in those situations, if you have engaged sexually with someone, you have to marry them, otherwise it angers the gods. The elder in the community had been involved in the safeguarding work for some time and he decided that they would do something different, sacrifice something else to the rain god and they would get the girl some support. So she wasn't forced to marry her brother. She was provided 
support, she was given counselling and connected with other people and she kept being involved in the programme for social support.

Clearly, conclusions cannot be drawn in terms of any causal relationships based on these data. However, the example illustrates how the organisational, personal and behavioural factors may work together to create a safeguarding culture through engaging with the wider community.

\subsubsection{Increased disclosures}

Participants unanimously reported an associated increase in the number of disclosures of safeguarding concerns in their organisation. This may be as a result of the implementation of relevant policy and procedures to manage cases, increased awareness and confidence in the system as well as an increase in the knowledge and skills of the people to whom disclosures were being made. One participant shared a similar experience to that described by many other participants. At the start of the project, the participant explained: "Within our organisation, in terms of formal protocols there are very few.” As such, Safeguard (2 (Procedures) had been rated as having nothing in place. This change to being fully in place by the end of the project, which represented a change in the organisational factors. Working towards the Safeguards had also changed the personal factors in terms of people's knowledge and feelings towards safeguarding, as explained by the same participant:

Now I can say that everyone knows that they need to adhere to the safeguards that are in place and sign the code of conducts for safeguarding children. People now know that there are procedures to be followed regarding safeguarding concerns.

This was viewed as a key contributor to the subsequent increase in disclosures: 
We have certainly seen behaviour change in terms of disclosures. This has been in two ways. Firstly, this has been through survivors coming forward. Before they did not have the language or confidence to speak. The Safeguards empower them and give them a voice. Secondly, other people are reporting concerns much earlier in the process. Before people may have thought, well, no one else is saying anything so I won't but now they have been validated.

Encouraging these disclosures and critically ensuring that appropriate support is in place for everyone involved should lead to the safeguarding of more children. This can be facilitated by the reciprocal relationships between the organisational factors (e.g., ensuring that clear and effective procedures are in place), personal factors (e.g., ensuring that people are aware of the procedures) and behavioural factors (e.g., ensuring that people are supported when concerns are disclosed). These mutually reinforcing relationships can thus help to build and maintain a safety culture.

Overall, this discussion has demonstrated how organisational, personal and behavioural factors can interact to influence the development and maintenance of a safety culture. Drawing on the work of Cooper (2000), it highlights the need to consider both the systemic and social factors (Parker, Lawrie, \& Hudson, 2006). It also highlights the fact that developing a safety culture is a dynamic and on-going process (Rhind et al., 2017). This is the first global project to apply the concept of safety culture to safeguarding in sport. This paper therefore makes a significant contribution through applying an innovative theoretical approach and a systematic research design that benefits from a comprehensive integration of mixed methods.

This research is particularly important due to the fact that the culture of sporting organisations is often discussed as a fundamental consideration when safeguarding children 
(Brackenridge et al., 2012). Rhind, Cook and Dorsch (2013) highlighted that the culture of an organisation plays a key role in preventing, mitigating or facilitating abuse. Furthermore, Mountjoy et al. (2016) discussed the potential for 'unhealthy' cultures to create inherent underlying threats to children. Tibbert, Anderson and Morris (2015) found that organisational cultures can be so influential that victims of maltreatment may become enculturated, rationalising and normalising maltreatment that they previously condemned.

If abuse in sport has a cultural basis, then so too should any safeguarding measures. Often efforts to safeguard children in sport have used approaches which either target the individual (e.g., educational workshops), interaction (e.g., codes of conduct) or the system (e.g., implementing policies and procedures). As abuse in sport is likely to take place due to the interaction between individual, interpersonal and systemic elements, the solution needs to be equally multi-facetted. Previous research suggests that a failure to do this has led to an over emphasis on human resource solutions (e.g., criminal background checks), which potentially undermines both the adult-child relationship in sport, and the positive aspects of children's participation (Piper, Garratt, \& Taylor, 2013).

The present research demonstrates that culture can be addressed through considering the organisational, personal and behavioural elements. It is only through targeting this range of factors that a comprehensive approach will be adopted, which can influence the fundamental values, assumptions and behaviours related to safeguarding in an organisation. This approach adopts a learning perspective to the study of culture and is fundamentally grounded in Bandura's (1986) Social Cognitive Theory. As such, behavioural change can occur through the reciprocal interactions between the person, the behaviour and the environment (discussed as the organisation in this paper). This raises important questions for managers in sport. In relation to the people within an organisation, do they know what they need to do in relation to safeguarding? In terms of the behaviour, what response do they experience when performing 
safe or unsafe behaviours? Are unsafe behaviours consciously or unconsciously promoted, such as concerns not being acted upon when disclosures are made? Are there opportunities for successful learning and are safe behaviours reinforced? In relation to the environment, does the organisation create conditions in which safe behaviours are conducive? Are policies, procedures and guidance readily available? Through addressing such questions, an organisation can work to develop and maintain an effective safety culture which safeguards children.

\section{Conclusion}

This research represents the first evaluation of a global strategy to safeguard children against abuse in sport. Overall, progress was identified at the organisational, personal and behavioural levels. The reciprocity between these factors was also perceived to facilitate the development and maintenance of a safety culture. This evidence suggests that the International Safeguards for Children in Sport represent an important resource for managing abuse in sport. The significant contribution of the present research is the shift in focus from understanding abuse in sport to understanding safeguarding in sport. This moves the field from a problem-focused approach to a solution-focused approach.

The relationships developed with the participants represented a strength of this research. It is likely that these relationships were an important vehicle through which the identified impact was achieved. The research was built on fundamental principles, which have subsequently been identified as best practice in facilitating impact. For example, Hanney, Greenhaldh, Blatch-Jones, Glover, \& Raftery (2017) identify three key strategies that are associated with achieving research impact on policy and practice. Firstly, there is collaboration with the end user to inform the planning of the study. This was certainly embedded in this project during the initial group meetings and online discussions through a philosophy of it being a coproduced project. Secondly, the project should be needs-led. The Safeguards project came out 
of a consensus of key organisations that there was a clear need for safeguarding in sport. Finally, there should be mechanisms in place for people to use the findings. This has been achieved through the International Safeguards being published along with the Implementation Guides. These strategies came about in a natural and organic way as opposed to being strategically planned. However, these characteristics do help to perhaps explain how this research has been able to achieve significant impact and the methods used can inform other researchers and end users as future projects are planned and conducted (Hanney et al., 2017). It is important to acknowledge that there are limitations associated with the approach adopted in this research. It is generally held that a level of engagement within the contexts that one wishes to study is useful in enhancing the researchers understanding. The authors made conscious efforts to build rapport with the participants. However, the relationships with these participants may have introduced bias into the data. For instance, at the start of the process, participants may have had concerns regarding the intentions of the research. Participants may have been apprehensive in terms of whether any information would be shared with funding agencies or the media. Efforts were made to allay these fears through written and verbal agreements, but these concerns still had the potential to impact the data. Later on in the project, rapport had been developed with the participants which may also have biased the data. The researchers were cognisant of these risks and emphasised throughout the need for honesty. As explained in the methods section, the authors took steps to maintain trustworthiness throughout the project. However, such limitations need to be acknowledged.

Future research is now merited with respect to the antecedents, further development and consequences of the Safeguards. In terms of antecedents, research is required into how key stakeholders in sport can be encouraged to adopt the Safeguards (e.g., Governing Bodies, Major Sports Events). Research can also explore the factors which influence the effective adaptation of the Safeguards to a given context (e.g., the size, purpose and location of an 
organisation). Whilst the present study involved 32 organisations from around the world, their remains great scope for the experiences of people working towards the Safeguards in diverse settings to be investigated. Finally, it is important to investigate the consequences of the Safeguards at various levels. This concerns children (e.g., well-being, participation and performance), the experiences of those around the children (e.g., parents and coaches) as well as the club/sport as a whole (e.g., organisational reputation and performance). The findings of this research should help to strengthen the rationale for safeguarding and ultimately promote the adoption and effective implementation of the Safeguards across global sport. This in turn should contribute to realising the principal behind the Safeguards; that any child should be able to participate in safe sport. 


\section{References}

Alexander K, Stafford A, Lewis R. 2011. The Experiences of Children Participating in Organized Sport in the UK. NSPCC: London.

Bandura, A. (1986). Social foundations of thought and action: a social cognitive theory. Englewood Cliffs, NJ: Prentice-Hall.

Brackenridge CH. 2001. Spoilsports: Understanding and preventing sexual exploitation in sport. Routledge: London.

Brackenridge, C. H., Kay, T., \& Rhind, D. J. A. (Eds.) (2012). Sport, Children's Rights and Violence Prevention: A source book on global issues and local programmes. London: Brunel University Press.

Brackenridge, C. H., Pawlaczek, Z., Bringer, J. D., Cockburn, C., Nutt, G., Pitchford, A., \& Russell, K. (2005). Measuring the impact of child protection through activation states. Sport, Education and Society, 10(2), 239-256.

Brackenridge, C. \& Rhind, D. J. A. (2014). Institutional responses to child abuse in sport: Reflections on 30 years of science and activism. Social Sciences. 3(3), 326-340.

Braun, V. \& Clarke, V. (2006). Using thematic analysis in psychology. Qualitative Research in Psychology, 3, 77-101.

Choudhry, R. M., Fang, D., \& Mohamed, S. (2007). Developing a model of construction safety culture. Journal of Management in Engineering, 23, 205-212.

Christian, M. S., Bradley, J. C., Wallace, J. C., \& Burke, M. J. (2009). Workplace safety: A meta-analysis of the roles of person and situation factors. Journal of Applied Psychology, 94(5), 1103-1127.

Chroni, S., Fasting, K., Hartill, M., Knorre, N., Martin, M., Papaefstathiou, M., Rhind, D. J. A., Rulofs, B., Toftegaard-Støckel, J., Vertommen, T., \& Zurc, J. (2012). Prevention of 
Sexual and Gender Harassment and Abuse in Sport: Initiatives from Europe and Beyond. Berlin: German Sports Youth.

Coalter, F. (2007). A Wider Role for Sport: Who's Keeping the Score? Abingdon: Routledge.

Cooper, M. D. (2000). Towards a model of safety culture. Safety Science, 36, 111-136.

Cox, S. J. \& Cheyne, A. J. T. (2000). Assessing safety culture in offshore environments. Safety Science, 34(1), 111-129.

Edwards, J. R. D., Davey, J., \& Armstrong, K. (2013). Returning to the roots of culture: A review and re-conceptualisation of safety culture. Safety Science, 55, 70-88.

Fernández-Muñiz, B., Montes-Peón, J., \& Vázquez-Ordás, C. J. (2007). Safety culture: Analysis of the causal relationships between its key dimensions. Journal of Safety Research, 38, 627-641.

Filho, A. P. G., Andrade, J. C. S., \& Marinho, M. M. D. (2010). A safety culture maturity model for petrochemical companies in brazil. Safety Science, 48, 615-624.

Frazier, C. B., Ludwig, T. D., Whitaker, B., \& Roberts, D. S. (2013). A hierarchical factor analysis of a safety culture survey. Journal of Safety Research, 45, 15-28

Hanney, S., Greenhaldh, T., Blatch-Jones, A., Glover, M. \& Raftery, J. (2017). The impact on healthcare, policy and practice from 36 multi-research project programmes: Findings from 2 reviews. Healthcare Research Policy and Systems, 26. Accessed on 30/8/17 at https://health-policy-systems.biomedcentral.com/articles/10.1186/s12961017-0191-yy.

Hartill, M., \& Lang, M. (2014). "I know people think I'm a complete pain in the neck": an examination of the introduction of child protection and "Safeguarding" in English sport from the perspective of national governing body safeguarding lead officers. Social Sciences, 3, 606-627. 
Hartill, M., Lang, M., \& Ashley, N. (2014). Safeguarding and child protection in rugby League. Olmskirk; Edge Hill University.

Jacobs, F., Smits, F., \& Knoppers, A. (2016). 'You don’t realize what you see!': The institutional context of emotional abuse in elite youth sport. Sport in Society, 20(1), 126-143.

Lindlof, T. R. \& Taylor, B. C. (2002). Qualitative Communication Research Methods. Thousand Oaks, CA: Sage.

Marshall, C. \& Rossman, G. B. (1999). Designing Qualitative Research. London: Sage. Mountjoy, M., Rhind, D. J. A., Tiivas, A. \& Leglise, M. (2016). Safeguarding the child athlete in sport: A review, a framework and recommendations for the IOC youth athlete development model. British Journal of Sports Medicine, 49, 883-6.

Papaefstathiou, M., Rhind, D. J. A. \& Brackenridge, C. (2012). Child protection in ballet: Experiences and views of teachers, administrators and ballet students. Child Abuse Review, 22, 127-141.

Parker, D., Lawrie, M., \& Hudson, P. (2006). A framework for understanding the development of organisational safety culture. Safety Science, 44, 551-562.

Patton, M. Q. (2015). Qualitative research and evaluation methods. London, UK: SAGE.

Piper, H., Garratt, D., \& Taylor, B. (2013). Child abuse, child protection, and defensive 'touch' in PE teaching and sports coaching, sport, education and society. Sport, Education and Society, 18(5), 583-598.

Raakman, E., Dorsch, K. \& Rhind, D. J. A. (2010). The Development of a typology of abusive coaching behaviours within youth sport. International Journal of Sports Science and Coaching, 5, 503-515.

Rhind, D. J. A., Cook, E. \& Dorsch, K. (2013). Looking at youth hockey from a human rights perspective. Sport in Society, 16, 254-266. 
Rhind, D. J. A., Kay, T., Hills, L., \& Owusu-Sekyere, F., (2017). Building a system to safeguard children in sport: The 8 CHILDREN pillars. Journal of Sport and Social Issues, 41, 151-171.

Rhind, D. J. A., McDermott, J., Lambert, E., \& Koleva, I. (2014). Managing safeguarding cases in sport. Child Abuse Review. 23, 418-426.

Sparkes, A. C., \& Smith, B. (2014). Qualitative research methods in sport, exercise and health. London, UK: Routledge.

Stoltenborgh, M., Bakermans-Kranenberg, M. J., Link, L. R. A. \& van Ijzenboorn (2015). The prevalence of child maltreatment across the globe. Child Abuse Review, 24, 37-50

Tibbert, S. J., Andersen, M. B., \& Morris, T. (2015). What a difference a "Mentally toughening" year makes: The acculturation of a rookie. Psychology of Sport and Exercise, 17, 68-78.

Tracy, S. J., Lutgen-Sandvik, P. \& Alberts, J. K. (2006). Nightmares, daemons and slaves: exploring the painful metaphors of workplace bullying. Management Communication Quarterly, 20, 148-185.

Vertommen, T., Schipper-Van Veldhoven, N., Wouters, K., Kampen, J. K., Brackenridge, C. \& Rhind, D. J. A. et al. (2016). Interpersonal violence against children in sport in the Netherlands and Belgium. Child Abuse and Neglect, 51, 223-236.

Wessells, M. G. (2009). What are we learning about protecting children in the community: An inter-agency review of the evidence on community-based child protection mechanisms in humanitarian and development settings. London: Save the Children. 
Table 1: A comparison of the self-audit data from the start and end of the project (Deliverers)

\begin{tabular}{|c|c|c|c|}
\hline & Nothing in place & Partially in place & Fully in place \\
\hline Developing Your & $15-0 \%$ & $22-11 \%$ & $63-89 \%$ \\
\hline \multicolumn{4}{|l|}{ Policy } \\
\hline Procedures for & $30-10 \%$ & $44-46 \%$ & $26-44 \%$ \\
\hline \multicolumn{4}{|l|}{ Responding to } \\
\hline \multicolumn{4}{|l|}{ Safeguarding Concerns } \\
\hline Advice and Support & $25-10 \%$ & $38-46 \%$ & $37-44 \%$ \\
\hline Minimising Risks to & $5-5 \%$ & $37-28 \%$ & $58-67 \%$ \\
\hline \multicolumn{4}{|l|}{ Children } \\
\hline Guidelines for & $13-0 \%$ & $50-22 \%$ & $37-78 \%$ \\
\hline \multicolumn{4}{|l|}{ Behaviour } \\
\hline Recruiting, & $35-5 \%$ & $18-39 \%$ & $47-56 \%$ \\
\hline \multicolumn{4}{|l|}{ Communicating and } \\
\hline \multicolumn{4}{|l|}{ Training } \\
\hline Working with Partners & $20-0 \%$ & $27-33 \%$ & $53-67 \%$ \\
\hline Monitoring and & $25-12 \%$ & $38-21 \%$ & $37-67 \%$ \\
\hline \multicolumn{4}{|l|}{ Evaluating } \\
\hline Overall & $21-5 \%$ & $34-31 \%$ & $45-64 \%$ \\
\hline
\end{tabular}


Table 2: A comparison of the self-audit data from the start and end of the project (Governors)

\begin{tabular}{|c|c|c|c|}
\hline & Not in place & Partially in place & Fully in place \\
\hline Developing Your & $35-0 \%$ & $27-0 \%$ & $38-100 \%$ \\
\hline \multicolumn{4}{|l|}{ Policy } \\
\hline Procedures for & $35-20 \%$ & $40-47 \%$ & $25-33 \%$ \\
\hline \multicolumn{4}{|l|}{ Responding to } \\
\hline \multicolumn{4}{|l|}{ Safeguarding Concerns } \\
\hline Advice and Support & $45-15 \%$ & $30-47 \%$ & $25-33 \%$ \\
\hline Minimising Risks to & $63-33 \%$ & $24-33 \%$ & $13-33 \%$ \\
\hline \multicolumn{4}{|l|}{ Children } \\
\hline Guidelines for & $25-0 \%$ & $37-50 \%$ & $38-50 \%$ \\
\hline \multicolumn{4}{|l|}{ Behaviour } \\
\hline Recruiting, & $50-10 \%$ & $25-23 \%$ & $25-67 \%$ \\
\hline \multicolumn{4}{|l|}{ Communicating and } \\
\hline \multicolumn{4}{|l|}{ Training } \\
\hline Working with Partners & $25-0 \%$ & $75-33 \%$ & $0-67 \%$ \\
\hline Monitoring and & $57-33 \%$ & $5-29 \%$ & $38-38 \%$ \\
\hline \multicolumn{4}{|l|}{ Evaluating } \\
\hline Overall & $42-14 \%$ & & $25-53 \%$ \\
\hline Overall & $42-14 \%$ & $33-33 \%$ & $25-53 \%$ \\
\hline
\end{tabular}

